\title{
Simultaneous Heating and Stirring by Magnetic Induction for Mixing of Carboxylic Acid Components and Hence Preparation of Binary Eutectic Mixtures
}

\author{
Sandeep Sharma ${ }^{1}$, Alok Kumar ${ }^{2}$, Vijendra Singh ${ }^{3}$ \\ ${ }^{1,2,3}$ Department of Chemistry; CCS University, Meerut (UP).
}

\begin{abstract}
Eutectic Mixtures had lowest possible melting point, so, their freezing point would also be lowest possible. In this way Eutectic Mixture had melting point/freezing point more close to room temperature as compared to their other compositions. This property of the eutectic mixtures as to get easily freeze did not allow the proper and uniform mixing of the components because when molten mixtures were stirred by stopping the supply of heat, they started freezing quickly, so, to made proper mixing to get exact melting point in an accurate and fast way, we applied magnetic induction heating method. This method provided simultaneous heating and stirring of the given mixture, which was energy saving, accurate and fast as well. In some acid-acid mixtures with their randomly selected compositions, simultaneous heating and stirring was performed, as a result it was found that the melting points were same every time and it was un-needed to take average of different melting points, as were obtained in earlier melting and then stirring techniques. As melting was proportional to high frequency and intensity of stirring was proportional to low frequency, so, providing multi-phase ac current, first at high frequency and then at low frequency the simultaneous melting and stirring was performed to achieve proper and uniform mixing of the acid-acid mixtures.
\end{abstract}

Keywords: Eutectic Mixture, Induction Heating, Multi-Phase AC Current.

\section{Introduction}

When we tried to prepare different compositions of two different carboxylic acids then their mixing by heating those particular acid components could just be achieved by heating first and then manual stirring of that molten mixture of acid-acid components ${ }^{1-2}$. The main problem arose when particular composition appeared to be "Eutectic Mixture", at that stage the mixture had minimum melting point and hence minimum freezing point, so, when this composition of eutectic mixture was heated then molten was obtained very easily but when the heating was stopped it started freezing with the same fast speed and ease, in this way the stirring of this eutectic mixture could not be done in a proper manner. If the molten form of eutectic mixture was further heated just to mix them properly then this additional heating could lead to decomposition of the mixture or even their magnetic or biological properties could be lost e.g. Malonic Acid suddenly decomposed after its melting point was reached ${ }^{3}$. Moreover, the continue supply of heat energy just to keep the mixture in the molten state to achieve uniform stirring was also an energy- wasting technique.

So it would be better if we stir the components of eutectic mixture while heating/melting of it. In this regard we had opted magnetic induction heating for the stirring and mixing of the two components of different compositions. It was the magnetic induction heating which could provide both heating and stirring simultaneously to form the uniformly mixed compositions. When single phase ac current was supplied then only heating was obtained but when multi-phase (Three-Phase) ac current was supplied then both heating and stirring could be achieved simultaneously. The frequency for providing simple heating was higher and frequency required for stirring was always lower than the frequency required for heating ${ }^{4-7}$.

If the given acid-acid composition was prepared by simple heating/melting followed by stirring then the mixing could not come out to be proper and uniform. It could be verified from difference in melting points of the same compositions in each melting point determination. Usually, average of three different melting points was taken. To solve this problem when we used simultaneous heating and stirring by magnetic induction for the mixing of carboxylic acid components ; then in each determination the melting point came out to be same for each composition (nevertheless condition of minimum human error is advised). This confirmed the proper and uniform mixing of acid- acid components in a single course of action. The other most important benefit of this method was that in normal heating/melting process heat was first of all supplied from the source to the container and then from the container to that substance which was to be melted, however, in magnetic induction heating method that particular container acted as the source of heat and in this way heat loss was minimized i.e. energy was saved. 


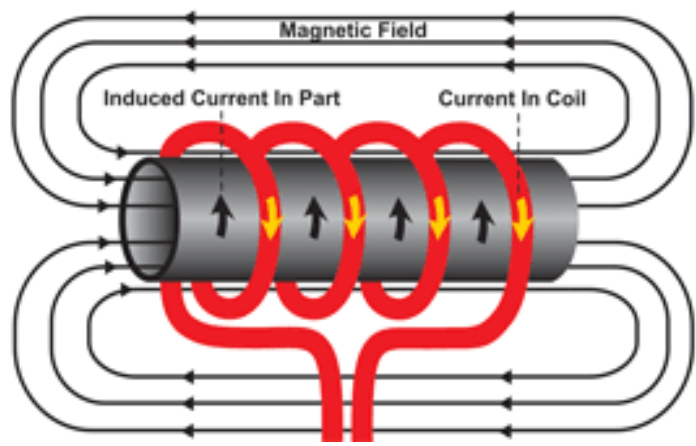

Fig.(1)

The combined action of the magnetic field and the electric currents creates electro-magnetic forces which raise the metal temperature and stir the melted metal. Magnetic materials naturally offer electrical resistance to the rapidly changing magnetic fields within the inductor. This resistance produces internal friction which in turn produces heat.

When an electric current $\mathrm{j}(\mathrm{x}, \mathrm{t})$ flows through a conducting body, whether solid or fluid, in the presence of a magnetic field $\mathrm{B}(\mathrm{x}, \mathrm{t})$, there is a force per unit volume ( Lorentz force)

$$
\mathrm{F}=\mathrm{j} \times \mathrm{B} \quad \ldots(1)
$$

That acts upon the conductor. In general, this force is rotational i.e. curl F. If the conductor is fluid, it cannot be compensated by a pressure gradient. In these circumstances, the fluid must move in response to the force. This, in its simplest terms, is the principle of electromagnetic stirring ${ }^{8-11}$.

\section{Experimental}

\section{TASK-I: Preparation of Different Acid-Acid Mixtures With Varying Compositions:}

We had taken different acid-acid mixtures with varying compositions so that melting point of different mixtures could be tested at different random compositions with the help of melting point apparatus.

\section{TASK-II: Heating/Melting and Stirring by Providing Multi-Phase AC Current:}

As multi-phase ac current provides simultaneous melting and stirring, so it had been used for the proper and uniform mixing of both the acid- acid components. In this process the container used for melting the given mixture should be ferromagnetic in nature like steel or iron. Resistivity of steel is higher due to the presence of tungsten and carbon in it, this high resistivity provides more heating of steel, that's why steel container was used. With increase in temperature resistivity increased and hence more heating was observed at high temperature.

TASK-III: To Determine The Melting Point of Different Acid-Acid Mixtures With Varying Composition:

Variable compositions were selected just to make it sure that for every composition, if uniform mixing was done then every time same melting point was obtained, which is shown in tables (1) to (4):

Malonic Acid-Benzoic Acid:

\begin{tabular}{|c|c|c|c|c|}
\hline Sr. No. & COMPOSITION & $\begin{array}{c}\text { MELTING POINT } \\
(\mathbf{K}) \\
(\mathbf{1})\end{array}$ & $\begin{array}{c}\text { MELTING POINT } \\
\mathbf{( K )}\end{array}$ & $\begin{array}{c}\text { MELTING POPINT } \\
(\mathbf{2})\end{array}$ \\
\hline 1. & $1: 9$ & 389.66 & 389.66 & 389.66 \\
\hline 2. & $9: 1$ & 395.16 & 395.16 & 395.16 \\
\hline
\end{tabular}

Table (1)

Succinic Acid-Benzoic Acid:

\begin{tabular}{|c|c|c|c|c|}
\hline Sr. No. & COMPOSITION & $\begin{array}{l}\text { MELTING POINT } \\
\text { (K) } \\
\text { (1) }\end{array}$ & $\begin{array}{l}\text { MELTING POINT } \\
\text { (K) } \\
\text { (2) }\end{array}$ & $\begin{array}{l}\text { MELTING POPINT } \\
\text { (K) } \\
\text { (3) }\end{array}$ \\
\hline 1. & $1: 4$ & 390.66 & 390.66 & 390.66 \\
\hline 2. & $4: 1$ & 444.66 & 444.66 & 444.66 \\
\hline
\end{tabular}


Table (2)

O-Salicylic Acid-Benzoic Acid:

\begin{tabular}{|c|c|c|c|c|}
\hline Sr. No. & COMPOSITION & $\begin{array}{c}\text { MELTING POINT } \\
(\mathbf{K})\end{array}$ & $\begin{array}{c}\text { MELTING POINT } \\
(\mathbf{K})\end{array}$ & $\begin{array}{c}\text { MELTING POPINT } \\
(\mathbf{K})\end{array}$ \\
\hline 1. & $2: 3$ & 394.16 & 394.16 & $(\mathbf{3})$ \\
\hline 2. & $3: 2$ & 411.16 & 411.16 & 394.16 \\
\hline
\end{tabular}

Table (3)

OxalicAcid-Benzoic Acid:

\begin{tabular}{|c|c|c|c|c|}
\hline Sr. No. & COMPOSITION & $\begin{array}{c}\text { MELTING POINT } \\
\mathbf{( K )}\end{array}$ & $\begin{array}{c}\text { MELTING POINT } \\
(\mathbf{1})\end{array}$ & $\begin{array}{c}\text { MELTING POPINT } \\
\mathbf{( K )}\end{array}$ \\
\hline 1. & $5: 5$ & 388.66 & 388.66 & 388.66 \\
\hline
\end{tabular}

Table (4)

So, every different acid-acid mixture with randomly selected composition was simultaneously melted and stirred for uniform mixing due to which each time we got same melting point which was not possible otherwise with other conventional methods.

\section{Results And Discussions}

In normal conventional methods when first heating/melting and then stirring was provided, then on each melting we used to get different melting point because in each melting some more mixing of the components of the mixture took place, which gave rise to different interactions in the mixture and hence a new melting point was observed but when same composition was uniformly mixed with simultaneous heating and stirring by magnetic induction then every time we got the same melting point, which showed that heating/melting first and then stirring could not provide a proper mixing due to which exact Eutectic Mixture was not formed but simultaneous heating/melting could provide fast and proper mixing of the acid-acid components. Hence this method was the best method for the preparation of Eutectic Mixture because in this method, due to proper and uniform mixing of components there were uniform interactions throughout the mixture and hence melting point came out to be same and more exact. So, mixing of acid-acid components by simultaneous heating and stirring by magnetic induction was the fast, accurate and energy saving method for the preparation of Eutectic Mixtures.

\section{Conclusion}

If in a mixture its two components say ' $A$ ' and ' $B$ ' were not uniformly mixed then three different types of interactions like A-A, B-B and A-B were present, each of these had its own melting point. Hence melting point range was larger. However, on each melting some more mixing took place and we got different melting point every next time. However when we provided the simultaneous heating and stirring then mixing was almost completed, so, mainly one type of interactions (A-B) were present and on further heating/melting again and again could not provide more uniform mixing and hence melting point came out to be same each time. While concluding, we could say that if mixing of the two acid components were proper with simultaneous heating and stirring by magnetic induction heating then their melting points were same each time. In this way we could prepare Eutectic Mixtures of different components with more accuracy and certainty, moreover this method was energy saving and fast as well.

\section{References}

[1] B.L. Sharma, N.K. Sharma,P.S. Bassi, Cryst. Res. Tekhnol, 18 (1983),peges 1191-1197.

[2] T.A. Mirnaya, A.P. Polishchuk, Y.V. Bereznitski, J. Chem. Eng. Data 1996, 41,pages 1337-1339.

[3] N. Weiner, "Malonic acid", Org. Synth.; Coll. Vol. 2: 376.

[4] S. Llorente, F. Monterde, J.M. Burdio, J. Acero, (2002). "A comparative study of resonant inverter topology used in induction cookers". 2009-05-20. 
[5] W. C.Moreland, The Induction Range: Its Performance and Its Development Problems, IEEE Transactions on Industry Applications, vol. TA-9, no. 1, January/February 1973 pages 81-86.

[6] Fairchild Semiconductors (2000-07). "Induction Heating System Topology Review". 2009.

[7] V. Rudnev, Handbook of Induction Heating CRC Press, 2003, page 92.

[8] H. K. Moffatt, Department of Applied Mathematics and Theoretical Physics, University of Cambridge, Silver Street, Cambridge CB39EW, England. 1990.

[9] S. Dong-Hwi ,E. Hyeju and P. Keun, Application of high-frequency induction heating to high-quality injection molding, in Plastics Engineering Annual Techncial Confernce Proceedings ANTEC 2010, Society of Plastics Engineers, 2010.

[10] R. Fields, Restaurant Success by the Numbers: A Money-Guy's Guide to Opening the Next Hot Spot, Random House of Canada, 2007, pages 144-145.

[11] H. Bach, D. Krause, Low thermal expansion glass ceramics, Springer, 2005,page 77. 